\title{
Dynamics and structure formation in thin polymer melt films
}

\author{
Ralf Seemann ${ }^{1}$, Stephan Herminghaus ${ }^{1}$, Chiara Neto ${ }^{2}$, \\ Stefan Schlagowski ${ }^{3}$, Daniel Podzimek ${ }^{4}$, Renate Konrad ${ }^{4}$, Hubert Mantz ${ }^{4}$ \\ and Karin Jacobs ${ }^{4}$ \\ ${ }^{1}$ Max-Planck-Institut for Dynamics and Self-Organization, Bunsenstraße 10, 37073 Göttingen, \\ Germany \\ 2 Department of Applied Mathematics, Australian National University, Canberra ACT 0200, \\ Australia \\ ${ }^{3}$ Department of Applied Physics, University of Ulm, Albert-Einstein-Allee 11, 89069 Ulm, \\ Germany \\ ${ }^{4}$ Experimental Physics, Saarland University, 66041 Saarbrücken, Germany \\ E-mail: k.jacobs@physik.uni-saarland.de
}

Received 24 November 2004

Published 18 February 2005

Online at stacks.iop.org/JPhysCM/17/S267

\begin{abstract}
The stability of thin liquid coatings plays a fundamental role in everyday life. We studied the stability conditions of thin ( 3 to $300 \mathrm{~nm}$ ) liquid polymer films on various substrates. The key role is played by the effective interface potential $\phi$ of the system air/film/substrate, which determines the dewetting scenario in case the film is not stable. We describe in this study how to distinguish a spinodal dewetting scenario from heterogeneous and homogeneous dewetting by analysing the emerging structures of the film surface by e.g. Minkowski measures. We also include line tension studies of tiny droplets, showing that the long-range part of $\phi$ does affect the drop profile, but only very close to the three phase boundary line. The dynamic properties of the films are characterized via various experimental methods: the form of the dewetting front, for example, was recorded by scanning probe microscopy and gives insight into the boundary condition between the liquid and the substrate. We further report experiments probing the viscosity and the glass transition temperature of nm-thick films using e.g. ellipsometry. Here we find that even short-chained polymer melts exhibit a significant reduction of the glass transition temperature as the film thickness is reduced below $100 \mathrm{~nm}$.
\end{abstract}

(Some figures in this article are in colour only in the electronic version) 


\section{Introduction}

Thin liquid films on solid surfaces are present in everyday life, though usually noticed only in passing. These films often act as lubricants, such as the oil film on the piston of a car engine or the polymer film on top of a hard disk. Thin liquid films are also used as coatings, e.g. in order to protect hardware from rust, to colour surfaces, or to change the wetting properties of substrates. The latter is particularly important for paper, which otherwise would be smeared during writing or printing. The tear film in our eyes wets and nourishes the cornea and with a blink dust gets wiped away. Thin liquid films can also change adhesion properties, a fact which becomes fairly obvious if one tries to build a sandcastle out of dry sand.

In all of these examples it is of great importance that the liquid wets the substrate. Only then can a homogeneous film be achieved. What if the film does not wet the substrate? Varnish painted onto a dusty or oily substrate, before it has had time to cure, will bead up into droplets and 'dewet' the substrate. Industry is thus often interested in preventing dewetting, whereas basic research is seeking for general rules to understand the origin of the phenomenon; of course, the latter is, in the long run, an indispensable precondition for the former.

\section{Fundamentals of wetting and dewetting}

In a Teflon ${ }^{\odot}$ coated frying pan one can achieve a stable oil layer only if one pours enough oil into the pan. Here the thick oil film is stabilized by gravity. As soon as the oil film is thinner than a certain thickness, namely the capillary length $\lambda_{\text {cap }}$ of the liquid, molecular forces dominate over gravitational forces and the film may dewet. The capillary length is given by $\lambda_{\text {cap }}=\sqrt{\sigma_{\mathrm{lv}} / \rho g}$ (for olive oil $\lambda_{\text {cap }} \approx 1.7 \mathrm{~mm}$ ), where $\sigma_{\mathrm{lv}}$ is the liquid/vapour surface tension and $\rho$ the density of the liquid. Hence dewetting of liquid films will only occur for 'thin' films, i.e. films thinner than $\lambda_{\text {cap. }}$.

Under which conditions is such a thin liquid layer stable or unstable on top of a substrate?

\subsection{Young's equation}

When a drop of a liquid is put on top of a solid surface, there are two competing effects. The interactions with the solid substrate make it energetically favourable for the drop to spread such that it wets the surface. However, spreading increases the area of contact between the liquid and vapour, which also increases the surface energy between the drop and the vapour. When the interaction with the solid surface dominates, one gets complete wetting, and when the surface tension term dominates, one gets 'partial wetting' as sketched in figure 1 [1-6].

For partial wetting one defines a contact angle $\theta$, which is given in equilibrium by a balance of macroscopic forces:

$$
\cos \theta=\frac{\sigma_{\mathrm{sv}}-\sigma_{\mathrm{sl}}}{\sigma_{\mathrm{lv}}}
$$

This is the famous Young equation [1] of 1805, where $\sigma_{\mathrm{sv}}$ and $\sigma_{\mathrm{sl}}$ are the solid/vapour and solid/liquid interfacial free energies, and $\sigma_{\text {lv }}$ is the liquid/vapour surface tension.

For $\theta=0$, a droplet will spread on the substrate. This case is termed complete wetting (figure 1(a)). For $0<\theta<\pi$ one speaks of partial wetting (figure 1(b)), and for $\theta=\pi$ of non-wetting (figure 1(c)).

In Young's equation, the contact angle is determined by the free energies of interfaces between semi-infinite media. In the case of long-range interactions that diverge as the film thickness decreases-e.g., van der Waals interactions - the 'energies of contact' can be modified. In other words, the equilibrium contact angle does not contain specific information 


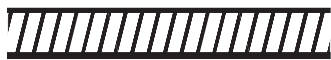
complete wetting

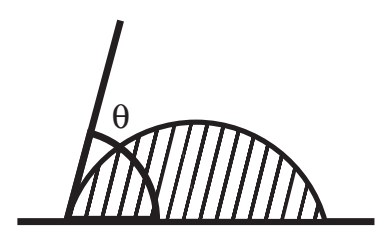

partial wetting

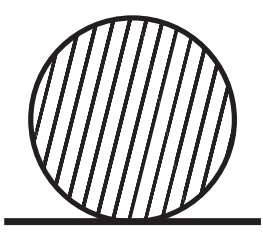

nonwetting

Figure 1. A sketch of a liquid drop on top of a solid substrate. Complete wetting is characterized by a contact angle $\theta=0$, partial wetting by $0<\theta<\pi$, and non-wetting by $\theta=\pi$.

about the (long-range) conjoining and disjoining forces acting on a thin liquid film. In order to predict stability conditions for a liquid layer, however, it is necessary to take these forces into account. Their dependence on the film thickness determines the way in which the uniform film is transformed into its equilibrium state, which consists of droplets of contact angle $\theta$ on the surface [7].

For example, a droplet of photoresist on a semiconductor may exhibit an equilibrium contact angle $\theta=20^{\circ}$ and therefore wets the surface only partially. What does this mean for a photoresist film that was prepared by a non-equilibrium technique (spin coating) on top of the semiconductor? According to Young's equation, the film is not stable. How will the film decay into droplets and at what speed will this process take place at a given temperature? Will there be time to cure the photoresist before it dewets? Depending on the thickness of the photoresist, the answers will be different, despite the fact that the contact angle $\theta$ which is finally formed does not change.

Macroscopic and molecular terms describing stability conditions were often mixed up and have led to confusion. It was one of the aims of this work to experimentally reconcile the macroscopic view of Young with the molecular forces acting on the liquid layer. The two following subsections show which forces are to be considered and how dewetting can be prevented to some degree by such interactions.

\subsection{Relevant interactions}

In this section the pair interaction between two atoms or small molecules is related to the interaction between a molecule and a flat surface and that between two flat surfaces.

2.2.1. Molecule-surface interaction. The total interaction between a molecule and a flat surface can be obtained by a summation over all molecule-molecule interactions if simple additivity of forces can be assumed. If the distance from an atom to the surface is large compared to the molecular diameter, the summation may be replaced by a triple integration.

Let us assume that the pair potential between atoms or small molecules is purely attractive and of the form

$$
w(r)=-C / r^{n}
$$

where $C$ is a constant and characterizes the strength of the interaction and $r$ is the distance between the two atoms or molecules. The triple integration has the effect of changing the dependence on $r$ and of introducing the quantity $\rho$, the number density of atoms in the solid. The net interaction energy for a molecule at a distance $r$ away from the surface will therefore be [3]

$$
w(r)_{\text {atom-surf. }}=\frac{2 \pi C \rho}{(n-2)(n-3) r^{n-3}} .
$$


2.2.2. Surface-surface interaction. Let us now calculate the interaction energy of two planar surfaces a distance $r$ apart. For two infinite surfaces, the result will be infinity, and so we have to consider the energy per unit surface area. Let us start with a thin sheet of molecules of unit area and thickness $\mathrm{d} z$ at a distance $z$ away from an extended surface of large area. From equation (3) the energy of interaction of this sheet with the surface is $w(r)=2 \pi C \rho(\rho \mathrm{d} z) /(n-2)(n-3) z^{n-3}$. Thus for the two surfaces, we have [3]

$$
W(r)_{\text {surf.-surf. }}=\frac{2 \pi C \rho^{2}}{(n-2)(n-3)(n-4) r^{n-4}} .
$$

The system used in the experiments, a non-polar polymer layered between an amorphous silicon oxide surface and air (vacuum), was chosen such that there are only two relevant types of interaction:

Steric repulsion is of relevant order within a few ångströms only and the resulting force is therefore termed 'short-range force'. The repulsion is due to overlapping electron shells and is typically described by a higher-order polynomial function and varies as $1 / r^{12}$. Considering two planar surfaces, this repulsion yields an interaction energy varying as $1 / r^{8}$ (equation (4)).

van der Waals forces arise from polarization fluctuations in the medium and occur in all materials. The microscopic van der Waals energy between two molecules, arising from the induced dipole-dipole interaction, varies as $1 / r^{6}$ in the static approximation, $r$ being the separation of the molecules. For larger distances $(>100 \mathrm{~nm})$ retardation effects have to be considered and the interaction falls as $1 / r^{7}$. Considering again two planar surfaces, the non-retarded interaction yields $[3,8]$

$$
W(r)=-\frac{A}{12 \pi r^{2}},
$$

where $W(r)$ is the energy per unit area and $A$ is known as the Hamaker constant [9] and is equal to

$$
A=\pi^{2} \rho_{1} \rho_{2} C .
$$

Here $\rho_{1}$ and $\rho_{2}$ are the number densities of the two surfaces involved.

The assumption of simple pairwise additivity inherent in the definition in $A$ of equation (6) ignores the influence of neighbouring atoms on the interaction between any pair of molecules. While definitely not correct or exact for a condensed phase, this is the conventional assumption. However, it turns out that all of the expressions for the interaction energies, equations (3)-(5), remain valid even within the framework of continuum theories. The only thing that changes is the way in which the Hamaker constant is calculated $[3,10,11]$, namely from the optical properties of the materials involved [3]. For $A<0(A>0)$, the system can gain energy by enlarging (reducing) the distance $r$ between the surfaces. Due to the $1 / r^{2}$ dependence of the potential, the van der Waals forces are long-range forces and act significantly on distances up to about $100 \mathrm{~nm}$.

In stratified systems with more than one layer between the two half-spaces the exact calculation of the van der Waals potential is quite onerous, since all mutual interactions have to be considered.

\subsection{The effective interface potential}

The effective interface potential, $\phi(h)$, contains short- and long-range interactions and is defined as the excess free energy per unit area which is necessary to bring two interfaces from infinity to a certain distance apart. The 'distance' is called $h$ on account of identifying the distance between the two interfaces (solid-liquid and liquid-gas) with the thickness of the liquid layer between them. 


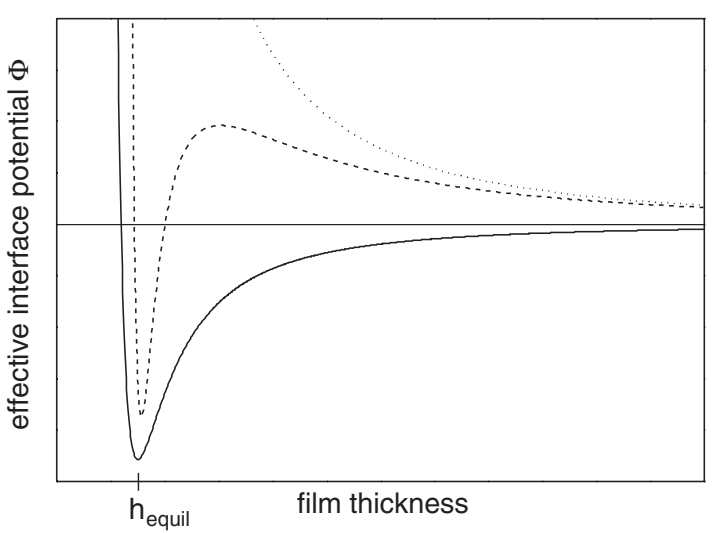

Figure 2. Effective interface potential $\phi$ as a function of film thickness. The dotted curve denotes the stable case, the dashed curve the metastable one, and the solid curve the unstable case.

In figure 2, three cases are shown, which are typical for the systems under consideration.

The dotted curve characterizes a film that is stable on the substrate, since energy would be necessary to thin the film. The equilibrium film thickness is infinite. The two other curves exhibit a global minimum of $\phi(h)$ at $h=h_{\text {equil }}$ and the system can gain energy by changing its present film thickness $h$ to $h_{\text {equil }}$. The solid curve characterizes a film that is unstable, whereas the dashed curve describes a metastable film. It is readily shown [12-15] that if the second derivative of $\phi(h)$ with respect to $h$ is negative, unstable modes exist whose amplitudes grow exponentially according to $\exp (t / \tau)$, where $\tau$ is the growth time that is characteristic for the respective mode. Furthermore, there is a characteristic wavelength $\lambda_{\mathrm{s}}$ of these modes whose amplitude grows fastest and which will therefore dominate the emerging dewetting pattern.

This process is analogous to spinodal decomposition of a blend of incompatible liquids which occurs if the second derivative of the free energy with respect to the composition is negative. There, as well, a certain wavelength exists whose amplitude grows the fastest. Following this analogy, dewetting via unstable surface waves has been termed 'spinodal dewetting' [16].

In the metastable case, for a film thickness where $\phi^{\prime \prime}(h)>0$, the system has to overcome a potential barrier in order to reach its state of lowest energy at $h=h_{\text {equil }}$. Some kind of nucleus, e.g. a dust particle, is required to lower $\phi(h)$ and can therefore induce dewetting. This rupture mechanism is termed 'heterogeneous nucleation' $[16,17]$. Close to the sign reversal of $\phi^{\prime \prime}(h)$ it is called 'homogeneous nucleation'. In this case, no nucleus is necessary because the thermal 'activation' is sufficient to overcome the energy barrier [18].

2.3.1. Linking the effective interface potential to macroscopic properties. As early as 1938, Frumkin stressed that there is a link between the effective interface potential and the macroscopic contact angle of Young [19]. In our nomenclature the relation reads

$$
\frac{\phi\left(h_{\text {equil }}\right)}{\sigma_{\mathrm{lv}}}=1-\cos \theta \text {. }
$$

Hence, if the surface tension $\sigma_{\text {lv }}$ is known, the global minimum of the effective interface potential $\phi\left(h_{\text {equil }}\right)$ determines the macroscopic contact angle $\theta$. 
Another 'macroscopically' accessible quantity that is linked to the effective interface potential is the spinodal wavelength $\lambda_{\mathrm{s}}[12,13]$ :

$$
\lambda_{\mathrm{s}}(h)=\sqrt{\frac{-8 \pi^{2} \sigma}{\phi^{\prime \prime}(h)}} .
$$

Equation (8) illustrates that only if $\phi^{\prime \prime}(h)<0$ (spinodal dewetting film) is $\lambda_{\mathrm{s}}$ real. For $\phi^{\prime \prime}(h)=0, \lambda_{\mathrm{s}}(h)$ diverges to infinity.

The above-mentioned links of the effective interface potential to macroscopic properties of the system are the reason that for a long time experimentalists were seeking for spinodally dewetting films. By the determination of $\lambda_{\mathrm{s}}$ as a function of $h$ one can gain insight into the course of $\phi^{\prime \prime}(h)$. By additionally measuring the equilibrium layer thickness $h_{\text {equil }}$ and the contact angle $\theta$, it is possible to reconstruct the complete effective interface potential. As an adjunct of the reconstruction of the effective interface potential $\phi$, the Hamaker constant $A$ of the system can be determined. The values for $A$ can be compared with the values calculated from optical properties of the media involved.

\section{Experimental details}

To study dewetting phenomena, a system was used that is on the one hand close to application cases (coatings, photoresist), yet on the other hand is easily controllable in the experiments. Polymers such as polystyrene (PS) are very suitable model liquids since they have a very low vapour pressure in the melt, and mass conservation is valid. Moreover, they are chemically inert, non-polar, and their dynamics can be tailored by choosing different chain lengths and annealing temperatures. Below the glass transition temperature $T_{\mathrm{g}}$, the film is glassy and can be stored for subsequent analysis. For this work, atactic polystyrene was used due to the known absence of any crystallization in this material.

For dewetting experiments it is necessary to prepare a thin liquid film in a non-equilibrium state on a substrate. Usually, a thin polymer coating is prepared from a solvent solution by one of the following standard techniques: spin coating, dip coating, and spraying. The solvent evaporates during the preparation procedure, leaving behind a smooth, glassy polymer layer. By spin coating a solution of polystyrene in toluene one can easily achieve a PS layer of thickness in the range of a few nanometres up to several micrometres. The roughness of the polystyrene layer is then similar to that of the underlying substrate. As substrates, freshly cleaved mica sheets, polished silicon ( $\mathrm{Si}$ ) wafers, and silanized Si wafers ('OTS-Si' 5 ) were used in our experiments. OTS-Si cannot be spin coated directly since the contact angle of the PS solvent on the substrate is too large and the entire droplet rolls off the surface. Consequently, polystyrene films were prepared on mica, floated onto a water surface, and picked up by the OTS-Si wafer. The thickness of the films was determined by ellipsometry; the topography of the layers was studied by optical microscopy and atomic force microscopy (AFM) in Tapping Mode $^{\mathrm{TM}}$. This operation mode ensures a reliable image of the soft surface.

To induce dewetting, the samples are heated above the glass transition temperature. Figure 3 shows a series of optical micrographs of an $80 \mathrm{~nm}$ thick PS film of molecular weight of $65 \mathrm{~kg} \mathrm{~mol}^{-1}$ ('PS(65k)') dewetting a silanized Si wafer. The series depicts a pattern formation process that is typical for most dewetting films, whether for a water film on a wax surface or a coating on a dusty substrate.

In the following sections, the process of dewetting is divided into three stages: in the early stage, holes are generated by a rupture process - cf figure 3(a); in the intermediate stage, the

5 Octadecyl-trichlorosilane; preparation details can be found in [20]. 

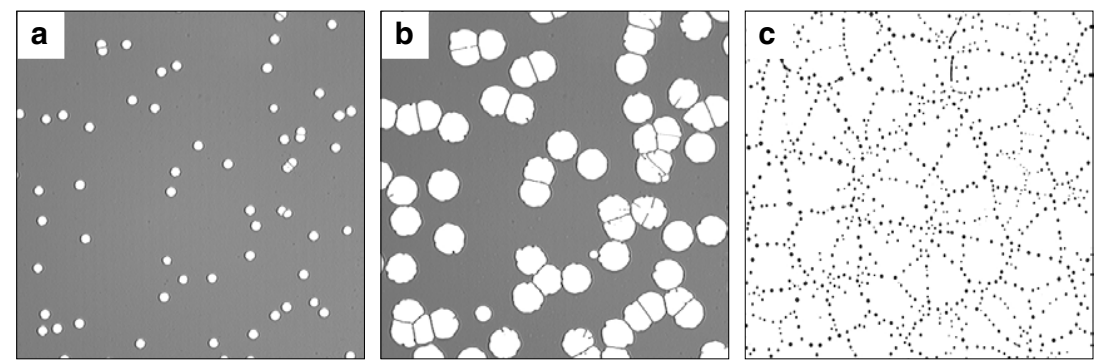

Figure 3. Pictures taken by a light microscope: a $80 \mathrm{~nm}$ thick polystyrene film of $65 \mathrm{~kg} \mathrm{~mol}^{-1}$ molecular weight is dewetting at $135^{\circ} \mathrm{C}$ from a hydrophobized silicon substrate. (a) $t=2 \mathrm{~min}$, (b) $t=18 \mathrm{~min}$, (c) $t=100 \mathrm{~min}$.

radius of the holes increases, leading to hole coalescence-cf figure 3(b). In the intermediate stage, the focus is on the dynamics involved in the dewetting process, and its impact on the hole profiles and on its influence on dewetting patterns. In the late stage, the straight ribbons that separate two coalescing holes decay into droplets due to Rayleigh-Plateau instability; cf figure 3(c).

\section{The early stage of dewetting: breaking the symmetry}

In the early stage, a metastable or unstable liquid film is transformed from a spatially homogeneous to a spatially inhomogeneous layer by the symmetry breaking process of dewetting. In this section, first the determination of the prevailing rupture mechanism will be introduced, and this is followed by a discussion of the properties of the respective mechanisms.

\subsection{The experimental distinction between nucleation and spinodal dewetting}

As pointed out above, spinodally dewetting films contain experimentally accessible information about the underlying forces and are therefore of special interest. Theoretically, the distinction between nucleation and spinodal dewetting was quite clear: Vrij [12] had already proposed in 1966 that a spinodal rupture of a free liquid film results in a dewetting pattern of 'hills and gullies' with a preferred distance $\lambda_{\mathrm{s}}$ after a certain time of rupture $\tau$. Experimentally, the rupture time $\tau$ is difficult to measure since the hole must have a certain size to be observable. Experimentalists instead concentrated on the evidence of a preferred wavelength $\lambda_{\mathrm{s}}$ in their systems.

If, however, the holes are randomly (Poisson) distributed, they are assumed to stem from heterogeneous nucleation, reflecting the fact that nuclei typically exhibit random statistics. The experimental distinction between spinodal dewetting and heterogeneous nucleation is far from being obvious. The thicker the films, the weaker the driving force, and the longer the growth time $\tau$ of the spinodal mode (typically, $\tau \propto h^{5}$ ). For thicker films, dewetting by heterogeneous nucleation may therefore be quicker and can suppress a spinodal pattern [21].

Moreover, chemical heterogeneities locally cause a change in $\phi$ and therefore the rupture conditions of the sample may vary from spot to spot leading to a less ordered dewetting pattern. This effect is more pronounced in thicker films due to the small driving forces and the large growth time $\tau$.

For dewetting patterns where the preferred wavelength cannot be detected by a Fourier transform (before the film rupture) or a radial pair correlation function $g(r)$, more powerful tools 
a)

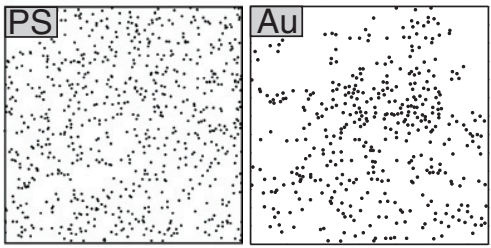

b) 2

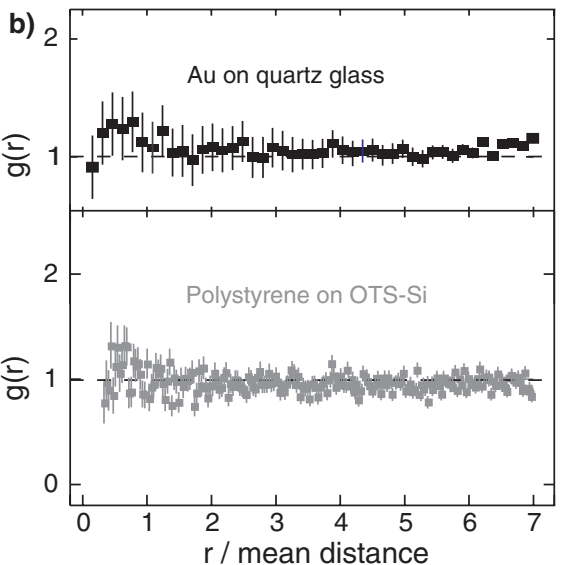

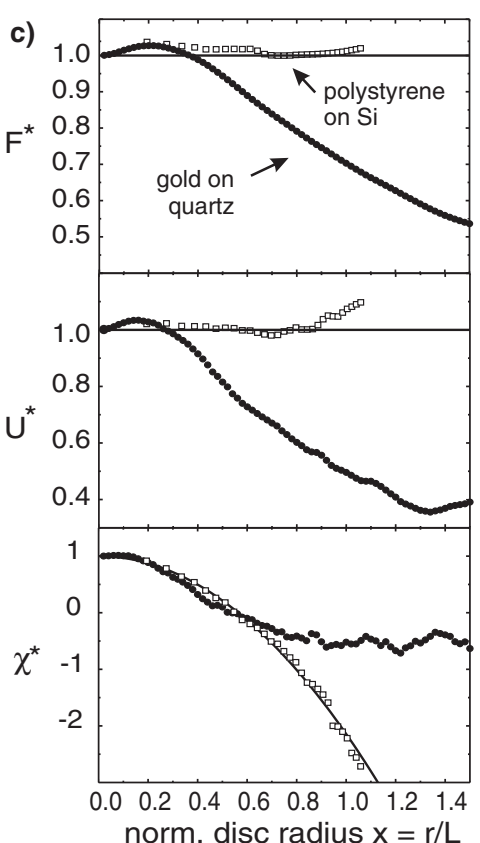

Figure 4. (a) Positions of holes in a PS film (left) and in a Au film (right). (b) The pair correlation function $g(r)$ of the Au and the PS film for the pattern shown. $r$ is given in units of the mean separation of objects. (c) Normalized morphological measures $F^{*}, U^{*}$, and $\chi^{*}$ of the Au (full circles) and of the PS film (open squares) as a function of the normalized radius $x, x=3 \mathrm{Dr} / L$, of the discs with mean separation $L$. The solid lines mark the expected behaviour for a Poisson point process. These images were taken from [25].

have to be applied. Here, Minkowski functionals - based on integral geometrical methodshave been shown to provide a versatile method for tracking down higher-order correlations [2224]. Their application to the experimental system of this work is described in detail in [25] in a comparative study on dewetting patterns of gold and of PS films.

The central idea is to determine the spatial statistics of the hole positions by adding a morphology to the point pattern [22]. This is done by assigning circular discs of radius $r$ to each of the hole positions. Due to possible overlap of the discs, the area $F$ and the boundary length $U$ of the set union of discs do not increase with $r^{2}$ and $r$, respectively. The larger the overlap, the slower the increase of the two measures. A third Minkowski functional, the Euler characteristic $\chi$, is a measure of the connectivity of a pattern and is defined in two dimensions as the mean curvature of the boundary line. For a random set of points, the dependence of $r$ for the three Minkowski functionals was analytically determined and then compared with the results obtained for the holes in the gold and in the PS films. The behaviour of the Minkowski measures for the holes in the PS films was shown to be in accordance with the result for a random set of holes (see figure 4). The Minkowski measures for the holes in the gold film, however, differed greatly from the curve of a 'random' sample, demonstrating the presence of correlations between the sites of the holes. This meant that the precondition for a spinodal mechanism was matched for the gold films, but not for PS films on the hydrophobized $\mathrm{Si}$ substrates in that study.

The method of determining the Minkowski measures of a point set was applied in all our studies whenever the presence of correlations between the sites of the holes could not be shown using a Fourier transform or a radial pair correlation function. 


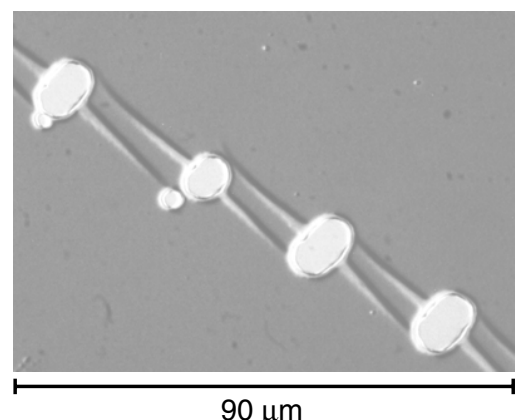

Figure 5. $60 \mathrm{~nm}$ thick PS(675k) film dewetting on an OTS-Si substrate. The folds are due to floating of the film during the preparation process. Preferred hole sites are in the vicinity of the folds where stress in the film is particularly high.

\subsection{Heterogeneous nucleation}

In the first experiments on the air/PS/Si system it was found that the average hole spacing scaled with the film thickness as expected for spinodal dewetting [26, 27]. Therefore, the scenario shown in figure 3(a) was often regarded as a typical spinodal dewetting scenario. However, it turned out later that this was not correct [24]. First of all, the typical timescale for dewetting does not fit the theoretical expectation. Secondly, as shown in [24] and [25], the hole arrangement indicates no spatial correlation, which is also in contradiction to theory. In both above-cited publications, the statistics of the hole positions was studied with the help of Minkowski functionals as described above.

Although it was later generally accepted that heterogeneous nucleation is the reason for the dewetting scenario shown in figure 3(a), the nature of the nucleus was still a puzzle. In some holes, by light microscopy or atomic force microscopy (AFM) an object could be observed right in the centre of a hole. Assuming the object to be a dust particle, the first approach was reducing the number of possible nuclei by improving the preparation conditions. The number of holes, however, could not be reduced significantly, which suggests that the physics of hole nucleation in polymer films may be deeper than a mere effect of 'dirt'. An example is shown in figure 5. Thus the possible cause of the occurrence of strain in the films induced by the preparation procedure was considered.

How can strain be induced by spin coating and how can it be released? A PS film is typically prepared by spin coating a toluene solution onto a smooth substrate. During spinning, the solvent evaporates and leaves the polymer behind as a thin layer on the surface. The process is still under discussion, but it is widely accepted that the radius of gyration $r_{\mathrm{g}}$ of the polymer in the thin film immediately after spin coating is larger than $r_{\mathrm{g}}$ for the PS melt [28]. Our hypothesis is that the rearrangement of chains in the thin film in order to achieve $r_{\mathrm{g}}$ for the melt is the reason for one class of nucleated holes.

To test this hypothesis, PS films were prepared on top of substrates that are wetted by PS, i.e. freshly cleaved mica sheets. After spin coating, the samples were annealed to a certain temperature $T_{\mathrm{a}}$ for successively longer times $t_{\mathrm{a}}$. Afterwards, the films were transferred to OTS-Si as described before and heated to a temperature $T_{\mathrm{d}}$ to induce dewetting. Then, the density of holes was recorded as a function of annealing time $t_{\mathrm{a}}$ on mica.

For PS(52k) for instance, for a film thickness of $60 \mathrm{~nm}$, one records an initial hole density $n_{\mathrm{i}}$ of $110 \mathrm{~mm}^{-1}$. After annealing for $t_{\mathrm{a}}=20 \mathrm{~min}$ to $T_{\mathrm{a}}=140^{\circ} \mathrm{C}$, a reduction of 40 per cent of the initial hole density was achieved. Annealing for longer times or to higher temperatures 
did not lead to a larger hole density reduction. The number density of nucleation sites which are deactivated or removed, $n_{\mathrm{s}}$, turned out to decrease with film thickness over more than four orders of magnitude. This suggests that there is some fundamental, well defined mechanism determining $n_{\mathrm{s}}$. For long-chained PS films, the percentage of reduction relative to the initial hole density was higher as compared to that for short-chained films, but significantly longer annealing times were necessary. Below the entanglement length, no reduction of the hole density could be observed. In order to check for effects due to possible residues of solvent, some series of experiments were done in a high-vacuum system (base pressure below $10^{-5} \mathrm{~Pa}$ ). Merely placing the samples under vacuum, no reduction of hole density could be observed. Also 'drying' of the samples overnight at temperatures slightly below the glass transition temperature, as practised by many other groups in order to reduce possible solvent content, did not yield a lower hole density.

It is possible to interpret this behaviour by assuming a stress field induced by chains that are not in their equilibrium conformation. The model can explain the scaling of $n_{\mathrm{s}}$ with film thickness and the influence of the chain length of the polymer. A more detailed description of the model is in preparation [29].

\subsection{Spinodal break-up}

After it was made possible to distinguish spinodal dewetting from nucleated break-up, the next step in the experiments was varying the driving forces in a controlled manner and looking out for a suitable experimental system where both rupture mechanisms could be studied. At the beginning of this work, only one system was known to dewet spinodally: thin gold films on top of quartz substrates [30]. Since these films were annealed by a laser pulse on a timescale of the order of ns, the deposited laser energy was not easy to control, and an online observation of the dewetting film was tried with pump/probe techniques, but proved difficult. Polymer films on top of silicon wafers, however, have proven to be an excellent model system. In contrast to metal films, polymer films such as polystyrene (PS) are dielectric and Hamaker constants can easily be determined from optical constants [3] on a 'two-interface system' such as air/PS/SiO ${ }^{6}$.

Silicon wafers with different oxide layer thicknesses were chosen as substrates. Here the problem of dealing with a 'three-interface system', air/PS/SiO/Si, is faced. In such stratified systems, equation (5) has to be modified and the long-range interaction of the system cannot be described using one Hamaker constant only.

Assuming additivity of forces, the system air/PS/SiO/Si may be described by summation of the van der Waals contributions of each of the single layers with thickness $h$ of the PS and $d$ of the $\mathrm{SiO}$ layer:

$$
\phi_{\mathrm{vdW}}(h)=-\frac{A_{\mathrm{SiO}}}{12 \pi h^{2}}+\frac{A_{\mathrm{SiO}}}{12 \pi(h+d)^{2}}-\frac{A_{\mathrm{Si}}}{12 \pi(h+d)^{2}},
$$

where $A_{\mathrm{SiO}}$ and $A_{\mathrm{Si}}$ are the Hamaker constants of the respective systems air/PS/SiO and air/PS/Si. Although not exact, this concept allows one to calculate the interaction energies even for stratified systems.

With the help of equation (9), the van der Waals potential of the experimental system is accessible, provided that the Hamaker constants can be calculated from the optical properties and the thickness of the $\mathrm{SiO}$ layer is known. As sketched in figure 6, the van der Waals potential gained from equation (9) can be varied with the thickness of the silicon oxide layer. From the course of the long-range part of the effective interface potential for a silicon substrate with a

6 In the following the term $\mathrm{SiO}$ stands for amorphous silicon (di)oxide. 


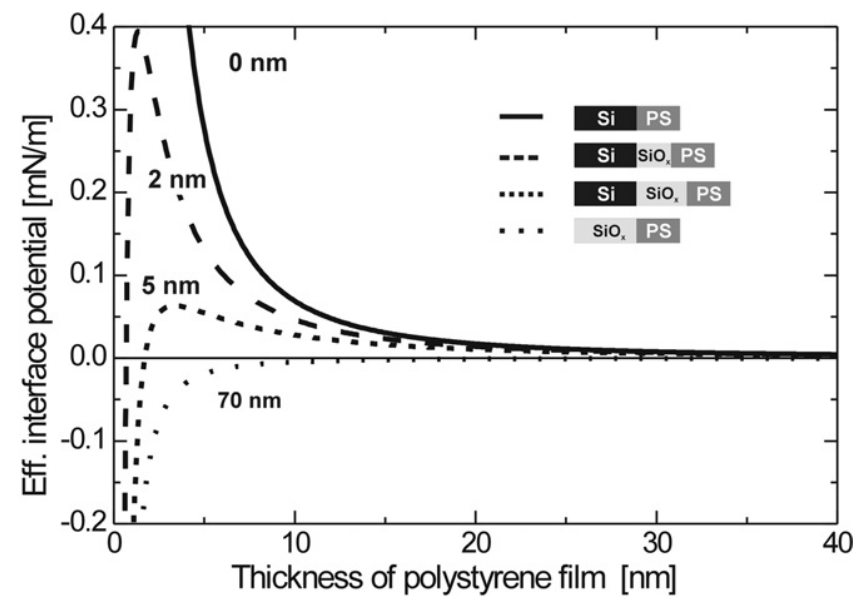

Figure 6. The long-range part of the effective interface potential for different $\mathrm{SiO}$ layer thicknesses, calculated with the formula given as equation (9). The Hamaker constants were calculated from the optical constants of the materials involved [3].

natural oxide layer thickness (about $2 \mathrm{~nm}$ ), shown in figure 6 as the dashed curve, it is obvious that PS films have to be prepared with a thickness well below $10 \mathrm{~nm}$ to enable the observation of the transition from the metastable to the unstable region.

Figure 7(a) presents a typical dewetting scenario for a thin PS(2k) film on a wafer with an oxide thickness of about $190 \mathrm{~nm}$ as observed by AFM: the formerly uniform film is transformed into having heaps and bumps all over. The pattern exhibits a characteristic length scale $\lambda_{\mathrm{s}}$, which can be determined e.g. via a Fourier transform, as depicted in the inset to figure 7(a). On increasing the prepared polymer film thickness, the dominant wavelength $\lambda_{s}$ increases, too, as expected for a spinodal dewetting scenario [12]. Experimental data for $\lambda_{\mathrm{s}}(h)$ are shown in figure 7(d) as the filled squares. X-ray reflectivity experiments revealed that the holes are not 'dry'. Instead, they are covered with a residual PS film of thickness $1.3 \mathrm{~nm}$. We interpret this thickness as the equilibrium film thickness $h^{*}$.

A preferred wavelength $\lambda_{\mathrm{s}}$ can also be found for PS(2k) films on wafers with an oxide thickness of 2.4(2) nm, but only for film thicknesses smaller than $4.1 \mathrm{~nm}$ (open circles in figure $7(d)$ ). Larger film thicknesses lead to dewetting patterns shown as examples in figure 7(c). Here, only a few holes are observed and they are at large distances from each other as compared to the holes in figure 7(a). This kind of dewetting pattern is known for thicker polymer films, and can be assigned to nucleated holes, as is seen from the absence of correlations in the hole positions [24]. The material that formerly covered the interior of the hole is accumulated in a surrounding rim which can be seen in the AFM scans as a bright ring.

On wafers with a natural oxide thickness of 1.7(2) nm, PS(2k) films smaller than $3.2 \mathrm{~nm}$ could not be prepared by spin coating from toluene solution; they dewetted during the spin coating process. Thicker films showed the same random pattern of holes as on wafers with $2.4 \mathrm{~nm}$ oxide thickness as depicted in figure 7(c).

By recording $\lambda_{\mathrm{s}}$ for different PS film thicknesses $h$, e.g. on top of Si wafers with a thick oxide layer, $\phi^{\prime \prime}$ can be calculated via equation (8). Fitting the second derivative of equation (5) to the data, the Hamaker constant $A_{\mathrm{SiO}}$ can be obtained as a fit parameter. For the reconstruction of $\phi(h)$, two further conditions have to be taken into account: the depth of the global minimum of $\phi$ is determined by the macroscopic contact angle $\theta$ via equation (7) and its position is fixed by the thickness of the equilibrium layer. In the present work, a short-range potential with a 


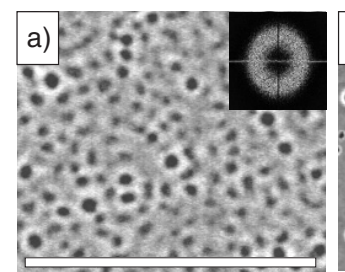

spinodal dewetting

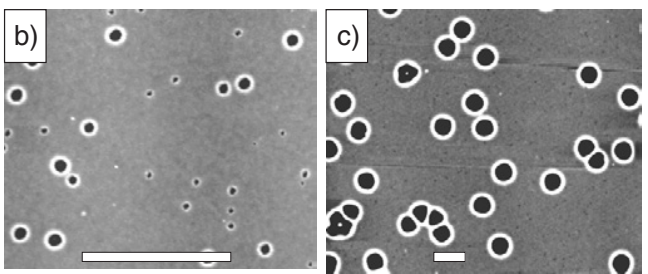

thermal nucleation heterog. nucleation
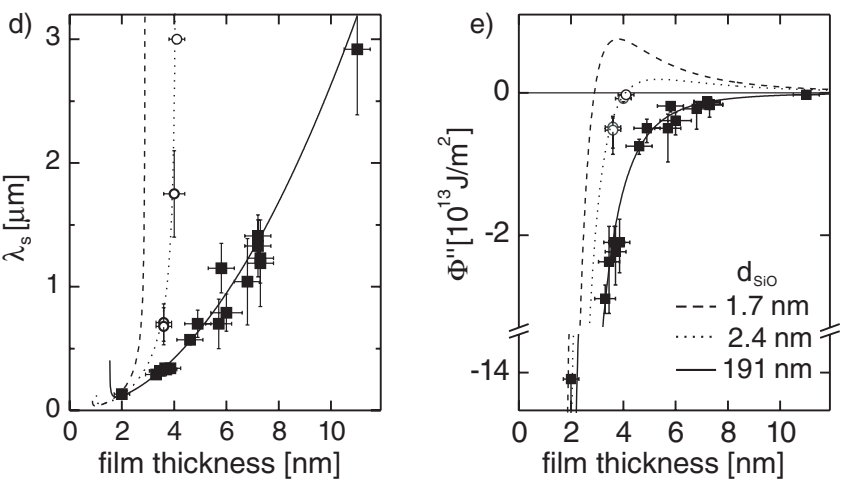

Figure 7. ((a)-(c)) AFM images of dewetting PS(2k) films. Scale bars indicate $5 \mu \mathrm{m}$; the $z$-scale ranges from 0 (black) to $20 \mathrm{~nm}$ (white). (a) $3.9 \mathrm{~nm}$ PS on a Si wafer with an oxide thickness of 191(1) $\mathrm{nm}$. The inset shows a Fourier transform of the image. (b) $4.1 \mathrm{~nm}$ PS on a wafer with an oxide thickness of 2.4(2) nm (c) $6.6 \mathrm{~nm}$ PS on a wafer with a 1.7(2) nm thick oxide layer. (d) Spinodal wavelength $\lambda_{\mathrm{s}}$ as a function of PS film thickness $h$ on a wafer with $2.4 \mathrm{~nm}$ oxide thickness (open circles) and a wafer with $191 \mathrm{~nm}$ oxide thickness (filled squares). (e) The second derivative of the effective interface potential $\phi^{\prime \prime}$ as a function of the film thickness $h$. These images were taken from [31].

$1 / h^{8}$ dependence, which is one of the forms commonly used in this context, was used to meet these conditions. A detailed description of the reconstruction of $\phi$, the fitting of the Hamaker constants, and the testing of the results can be found in [31] and [32].

Due to the knowledge of $\phi$, the wettability of the substrate can be tailored and a PS film can be stable, metastable, or unstable, depending on the PS and $\mathrm{SiO}$ layer thicknesses. In [32], this prediction is tested by performing further dewetting experiments and by reconstructing the effective interface potential from the dewetting patterns. There, a stability diagram is also developed. Moreover, the tailoring of a stratified system is tested not only by varying the thickness of the $\mathrm{SiO}$ layer, but also by applying thin layers of gold, $\mathrm{MgF}_{2}$, and OTS on top of the silicon substrates.

By investigating the onset of spinodal dewetting by in situ AFM scanning, the growth time $\tau$ for the spinodal wavelength can be determined. For the first time, a consistent picture is gained, comprising the timescale of dewetting, the Hamaker constants involved (as fitted and as calculated from optical properties), and the viscosity of the thin film.

\section{The intermediate stage: the growth of holes}

The dynamics of hole growth is only observable if the density of holes is not too high; they must have space to grow in a 'uniform' film before coalescing.

In the typical dewetting scenario, isolated holes pop up in an otherwise rather uniform film. This is true for heterogeneous break-up and in most of the cases for spinodal rupture. 


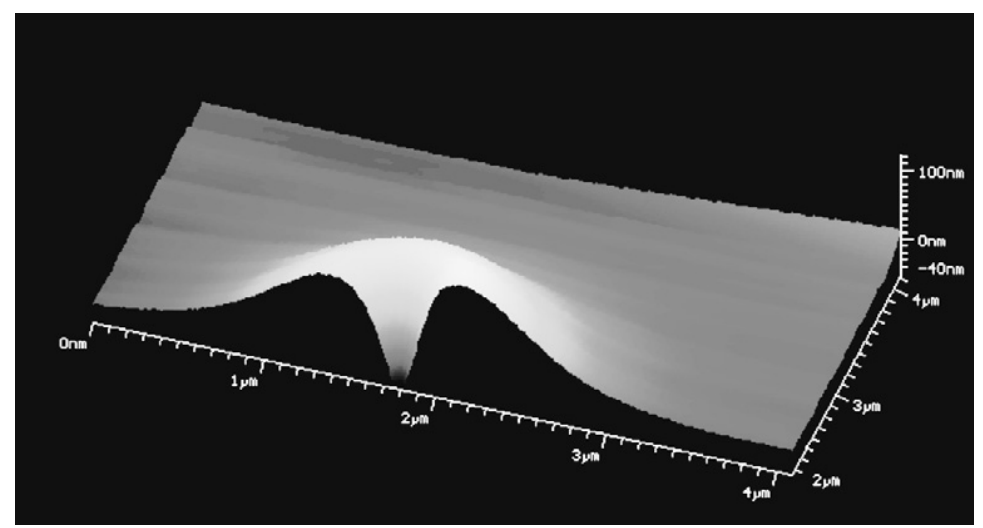

Figure 8. An AFM cross section of a hole in a PS(65k) film on OTS-Si. The material removed from the inside of the hole is accumulated in a characteristic rim surrounding the hole.

Only for extremely low film thickness is a 'crests and troughs' pattern morphology observable (figure $7(\mathrm{a})$ ).

How is a film with isolated holes transformed into its inverse, isolated droplets on top of a 'dry' substrate? Figure 3(b) shows the film of figure 3(a) at a later time. The material that formerly covered the inside of the hole is accumulated in a rim surrounding the hole. The liquid polymer forms a finite contact angle with the substrate, which is characteristic of a non-wetting system.

Figure 8 shows a three-dimensional cross section through a hole, observed by AFM. The three-dimensional shape of such a rim is influenced by properties of the materials involved, e.g. the contact angle of the liquid on the solid substrate and the chain length of the polymer.

\subsection{Dynamics of hole growth in two dimensions}

The driving force for the growth of the hole is a negative spreading coefficient $S$ [6] expressing the fact that the solid surface prefers to be dry:

$$
S=\sigma_{\mathrm{lv}}(\cos \theta-1) .
$$

For thick films, $S$ is balanced by gravity and a hole will only expand if the film thickness is smaller than a critical value. In our case, however, gravity is negligible and $-S$ is the driving force on the contact line. For the system air/PS/Si wafer, $S=-0.3 \mathrm{mN} \mathrm{m}^{-1}$ and for air/PS/OTS/Si wafer, $S=-15 \mathrm{mN} \mathrm{m}^{-1}$.

An ideal (Newtonian) liquid that flows has only one possible mechanism for dissipating energy: viscous friction. For a retracting rim, the site of highest shear rate is at the three-phase contact line. Since the contact angle stays constant during hole growth, $S$ is constant too, and the radius $R$ of the hole will grow with constant velocity.

An entangled polymer melt, however, does not flow like an ideal liquid. de Gennes [33] predicted that polymers slip on smooth, passive surfaces. The slippage is characterized by the extrapolation length $b$, defined as the distance from the wall at which the velocity extrapolates to zero. A pressure gradient parallel to the film induces the usual Poiseuille flow in thick films $(h \gg b)$ but a plug flow in thin films $(h<b)$ : the polymer slides like a solid and the viscous dissipation is confined at the liquid/solid interface [34-37]. The volume of the rim is determined by the conservation law for the liquid volume. Hence with the growing rim, the area of slippage at the liquid/solid interface increases too, leading to an $R \propto t^{2 / 3}$ dependence in the case of slippage [35]. 
a) no slippage: viscous flow

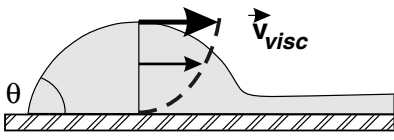

$\frac{\mathrm{dR}}{\mathrm{dt}}=\mathrm{v}_{\text {visc }}$, thus $\mathrm{R} \sim \mathrm{t}$ b) full slippage: friction at interface

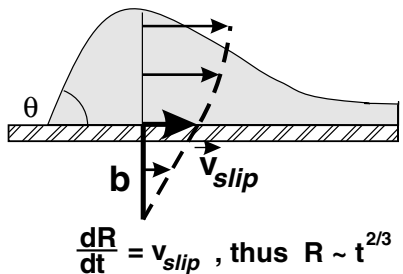

Figure 9. The two possible mechanisms of friction and the growth law of the holes; $R$ denotes the radius of a hole and $t$ the time.
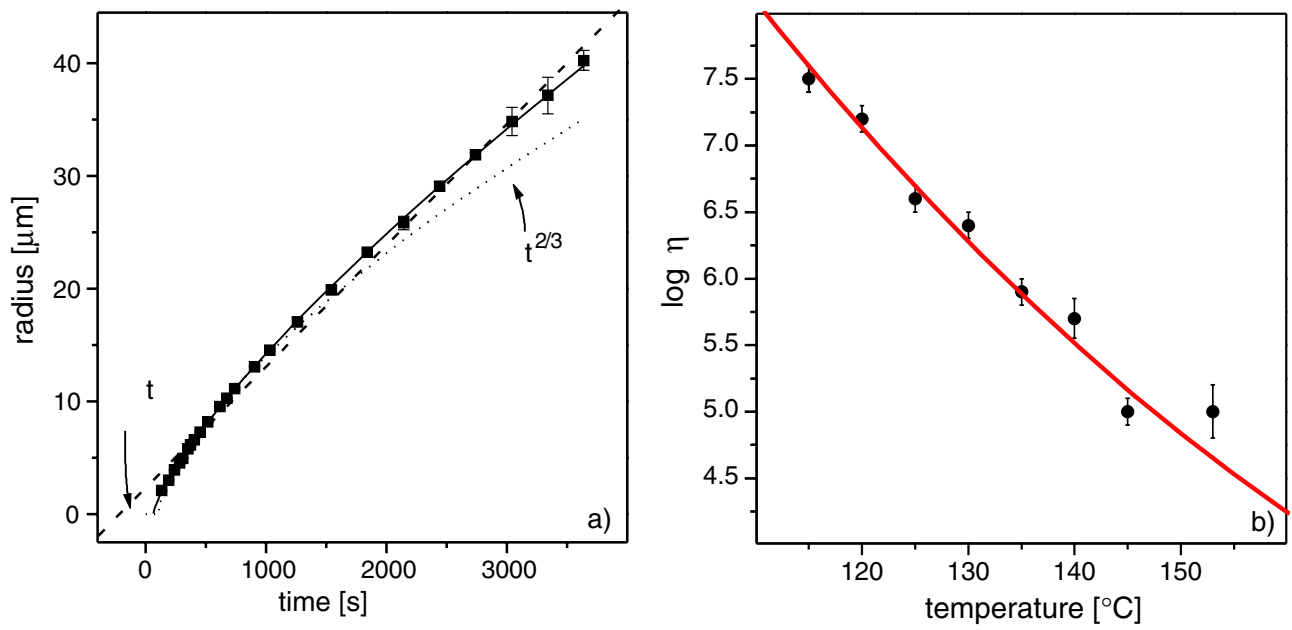

Figure 10. (a) Typical behaviour of the hole radius as a function of time. Dashed curve: the theoretical prediction for no-slip conditions. Dotted curve: the prediction for full slip, i.e., when dissipation takes place only by slippage of the film across the substrate. Solid curve: our theory fitted to the data, which allows us to extract the effective viscosity and the slip friction of the liquid film on the substrate. (Part (a) is taken from [40].) (b) Viscosity $\eta$ extracted fitting the function from [40] to the hole growth data of PS $(65 \mathrm{k})$ for various temperatures. The solid curve is a VogelFulcher curve for bulk viscosity. From the fitting function we can extract $\log \eta$ with a constant offset. When 'calibrated' with the bulk viscosity, the fitting function enables the measurement of $\eta$ with high precision.

A 'real' polymeric liquid, however, is expected to dissipate energy by both slippage and viscous flow. In a small rim (either for a tiny hole or for a rim in a very thin film), slippage plays the prominent role, whereas for a large rim, viscous dissipation dominates the hole growth. Therefore, a hole should first grow with a radius of $R \propto t^{2 / 3}$ in a 'strong slippage regime', and then shift to a no-slip regime, where $R \propto t[34,35]$.

It is common to fit a function $R(t)=a\left(t-t_{0}\right)^{b}$ to the data $[34,38,39]$ with an exponent $b$ ranging in $0.5<b<0.8$. This fitting function, however, gives different results for $b$ at the early and at the late stages of hole growth. To describe the entire growth law with a single fitting function, both friction mechanisms have to be considered simultaneously.

In [40], an expression for the temporal development of circular holes is derived, which correctly yields the limiting cases of pure viscous flow and full slippage of the film on the substrate. Good agreement between experiment and theory is found for the whole range of hole growth $[40,80]$, cf figure 10 . This growth law can be used to characterize the interplay 

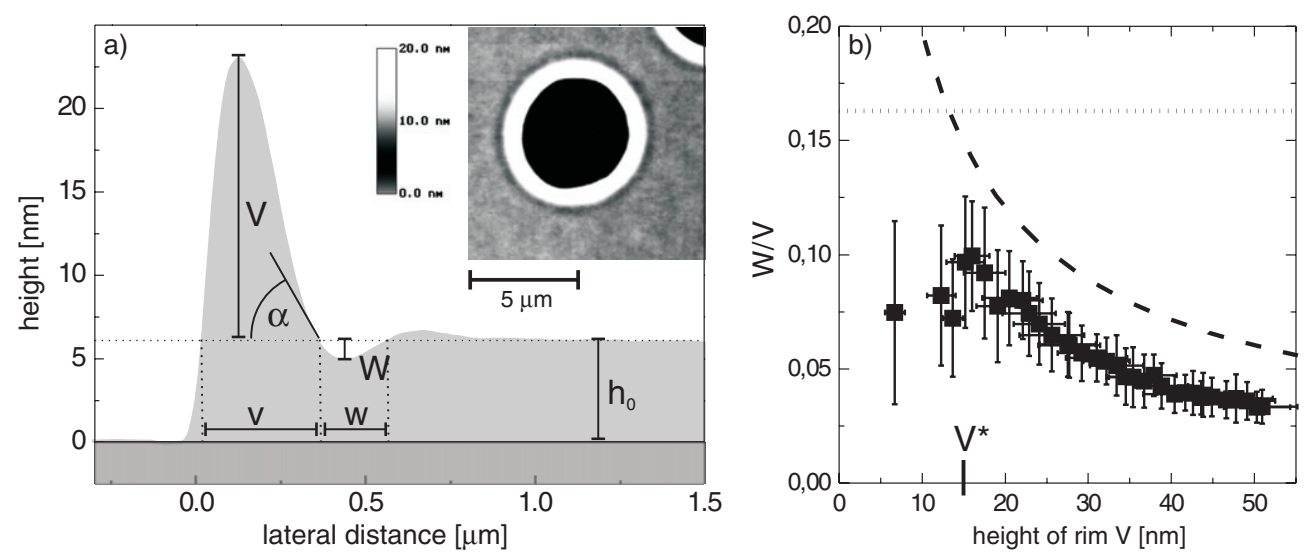

Figure 11. (a) Inset: an AFM scan of a hole in a 6.6(2) nm thick PS(2.24k) film on a silicon wafer with a $191 \mathrm{~nm}$ thick oxide, for $30 \mathrm{~min}$ at $T=80^{\circ} \mathrm{C}$. The large diagram shows a radial cross section of this hole, where $V$ describes the height of the rim, $W$ the depth of the depression with respect to the level of the unperturbed film. (b) The ratio $W / V$ for rim height $V$. Dots: experimental data for a 11.0(4) nm thick PS(2.05k) film on a Si wafer with $191 \mathrm{~nm}$ of oxide at a temperature of $T=90^{\circ} \mathrm{C}$. Dashed and dotted lines: the theoretical expectation in the lubrication approximation for the same system, gained from the analytic calculation without any elastic properties. Dotted line: the solution for the early stage, $W / V \approx 0.163$. Dashed line: the solution for the later stage, using the reduced viscosity $\eta=300 \mathrm{~Pa} \mathrm{~s}[73,74]$ with the boundary condition gained from experiment: $\alpha_{\text {theo }}=1.0^{\circ}$. These images were taken from [44].

of viscous flow and sliding friction. In that way, the rheology of a viscoelastic liquid can be probed on a nm scale; it is not accessible by standard rheological techniques.

\subsection{The morphology of the rim: dynamics in three dimensions}

A liquid rim surrounding a hole represents a disturbance in the otherwise uniform film. How does the shape of the rim - in the theorist's view basically a circular segment (figure 9(a)) change with system parameters such as temperature, chain length, dewetting velocity, and wettability of the substrate? How does e.g. slippage affect the profile?

With an AFM, the 3D rim profile can be monitored as depicted in figure 8. By means of a heated sample holder, in situ AFM scans were possible. During the growth of a hole, the contact angle $\theta$ at the three-phase contact line stays constant and is also invariant against temperature changes. Profiles of a hole taken at different times look quite similar: the profiles are typically asymmetric, as sketched in figure 9 (a) and, as can be seen in figure 8, at the 'dry' side of the profile - at the three-phase contact line-there is a higher slope than on the 'wet' side of the profile, where it meets the undisturbed film. As can be seen in figure 12, the asymmetry of the profile increases with increasing chain length, or, in other words, the 'dynamical wet contact angle' decreases and the profile is broadened.

For very small chain lengths, though, we found a peculiar behaviour of the profilecf figure 11-not yet seen in a real system [44]: at the 'wet' side of the rim, the melt 'dips' underneath the level of the unperturbed film, resulting in a kind of trough in the perimeter of the rim. Theoretically, this behaviour was found before by Safran and Srolovitz [41] and by Ghatak et al [42], yet the trough was suspected to be a numerical artifact [43]. If one assumes the liquid to be a Newtonian liquid, the profile can be described in terms of an exponentially decaying harmonic oscillation, taking the 'dynamical wet contact angle' as a boundary condition in the late state of hole growth. In that way, the observed depth of the trough, as a function of 


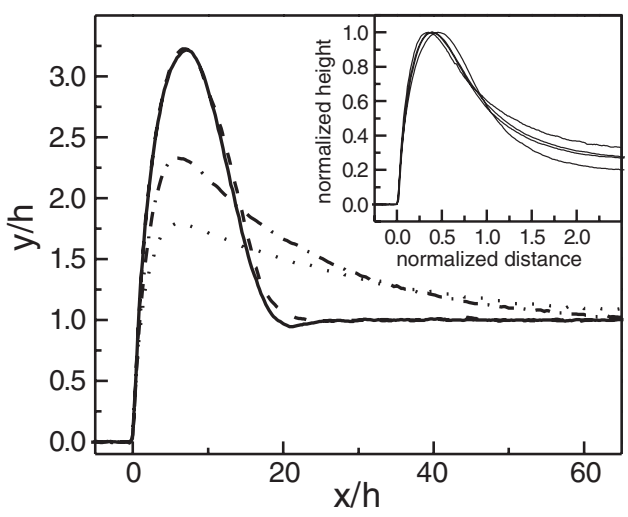

Figure 12. Cross sections as gained from AFM scans of holes in polystyrene films with different chain lengths on OTS-Si: solid curve: PS(2k); dashed curve: PS(18k); dot-dashed curve: PS(101k); dotted curve: PS(600k). The thickness $h$ of the films and the diameters of the holes are chosen to be about the same. Inset: hole profiles of PS $(65 \mathrm{k})$ taken at temperatures between 125 and $145^{\circ} \mathrm{C}$. Differences in film thickness and hole size lead to slight differences of the profiles. These pictures were taken from [44].

rim height, can be described qualitatively, but it is found to be roughly a factor of two smaller than the theoretical prediction. To account for the difference, further experiments have been performed with longer chain lengths of PS. The hypothesis is that even for a short-chain PS melt, viscoelastic properties come into play which substantially alter the flow behaviour and therefore the rim profile. (Viscoelastic liquids can respond to shear forces and this may result in sliding friction ('slippage') at the interface between the melt and substrate.) On increasing the chain length of a PS melt, not only are the viscoelastic properties enhanced, but also the viscosity is increased. To distinguish one effect from the other, the experiments shown in figure 12 were performed at different temperatures, which affects the viscosity to a known extent, but which hardly changes its viscoelastic properties. The experimental results show that it is not the viscosity that affects the profile. A general study of the rim shape and how it is affected by the viscoelastic effects, contact angle, and interfacial potential can be found in [78]. The effects of liquid slippage on the evolution of a spanwise morphology in the liquid rim are being further investigated [79].

\section{The late stage: droplets on the surface}

The late stage of dewetting in our terminology begins when a hole cannot grow any further since it meets neighbouring holes. Upon meeting ('coalescence'), the rims of the two holes overlap and form a straight common liquid ribbon, which in cross section is a portion of a cylinder. Such a liquid cylinder is dynamically unstable and decays via a Rayleigh-Plateau instability [45] into single droplets. The final stage is hence a polygonal network made up of droplets, as depicted in figure 3(c).

In equilibrium, these droplets should have the form of a spherical cap, fixed by Young's contact angle [1]. Young's equation, however, assumes that the contact angle is determined by the macroscopically measured surface tensions which may be modified in the case of longrange interactions. Hence, if the height of the droplets is in the range of the effective interface potential it is not clear whether or not their shape will still resemble a spherical cap [18, 46, 47]. AFM scans reveal, however, that even very small droplets do indeed exhibit the shape of a spherical cap [56]. 

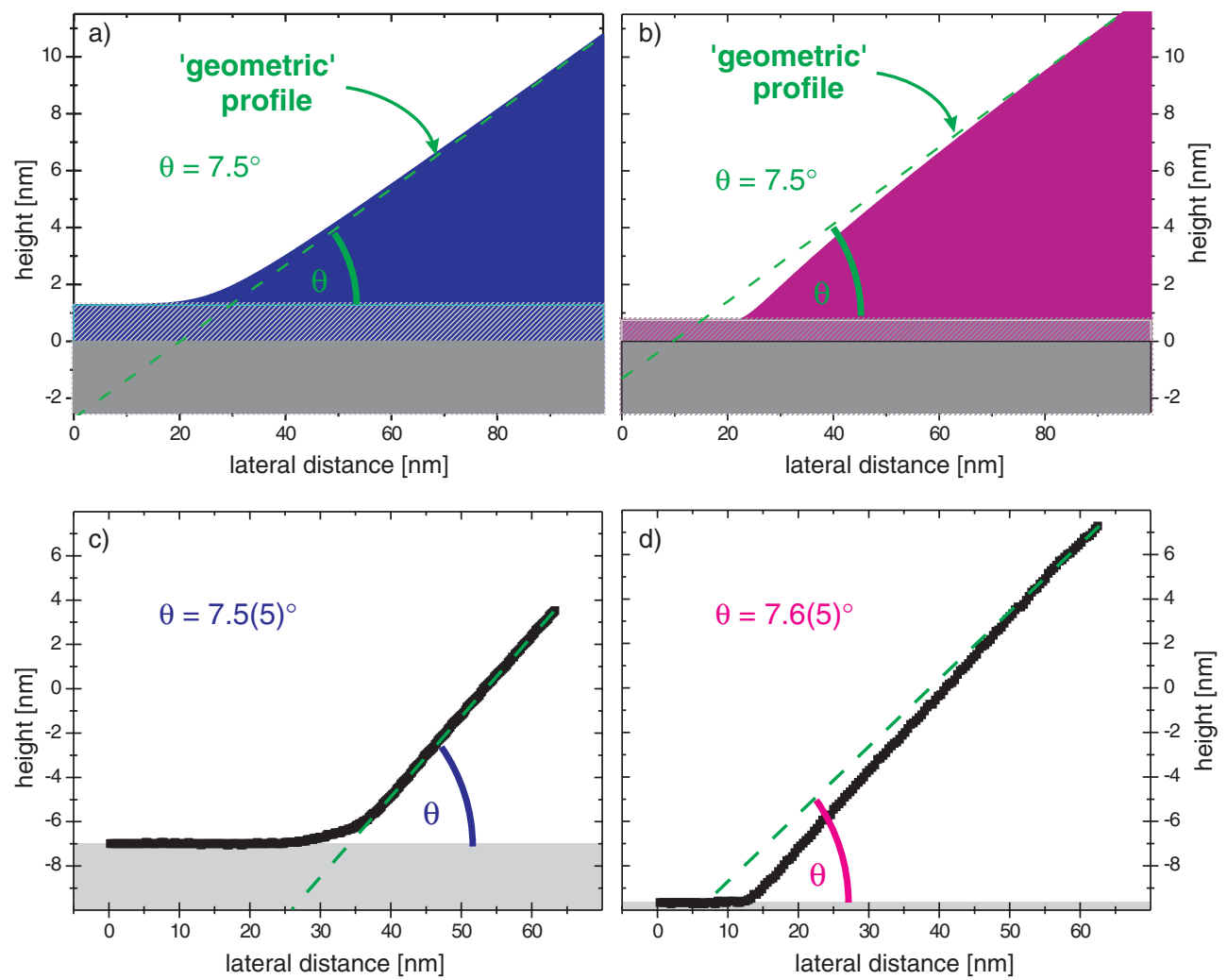

Figure 13. Droplet profiles at the three-phase contact line as calculated from the reconstructed effective interface potential from [31] ((a), (b)) and as measured at PS droplets by AFM ((c), (d)). ((a), (c)) PS on top of a thick (191 nm) Si oxide substrate. ((b), (d)) PS on top of a thin (1.7 nm) Si oxide substrate. The deviation from Young's contact angle is very small and is supposed to affect the droplet shape only in the nm range; the hatched area denotes the equilibrium PS layer with thickness $h_{\text {equil }}=1.3 \mathrm{~nm}$.

From the reconstructed effective interface potential $\phi$ in [31] the course of the droplet shape in the vicinity of the three-phase contact line can be inferred; cf figure 13. The deviation between the 'geometric' profile — as given by the Young's contact angle $\theta$ - and the 'real' profile occurs only very close (a few $\mathrm{nm}$ ) to the three-phase contact line. This corroborates the results from above, that the influence of the long-range interactions on the droplet shape should be negligible even for a very small droplet.

Up to now, the droplets under consideration have been viewed only in the form of a radial cross section, i.e. in 2D. Therefore, effects due to the curvature $\kappa$ of the three-phase contact line, which only come into play in 3D, were neglected. The so-called 'line tension' $v$, defined as the excess free energy per unit length of the contact line, is responsible for the fact that the contact angle of very small droplets may differ from the Young angle $\theta$ [48-51]. The difference is given by the modified Young equation

$$
\cos \theta_{\text {mod }}=\cos \theta-\frac{\nu \kappa}{\sigma_{\mathrm{lv}}} .
$$

Determining the curvature and the contact angle $\theta_{\text {mod }}$ of PS droplets of various sizes, the line tension can be inferred [52-55, 47]. Our results for the PS droplets shown in figure 14 

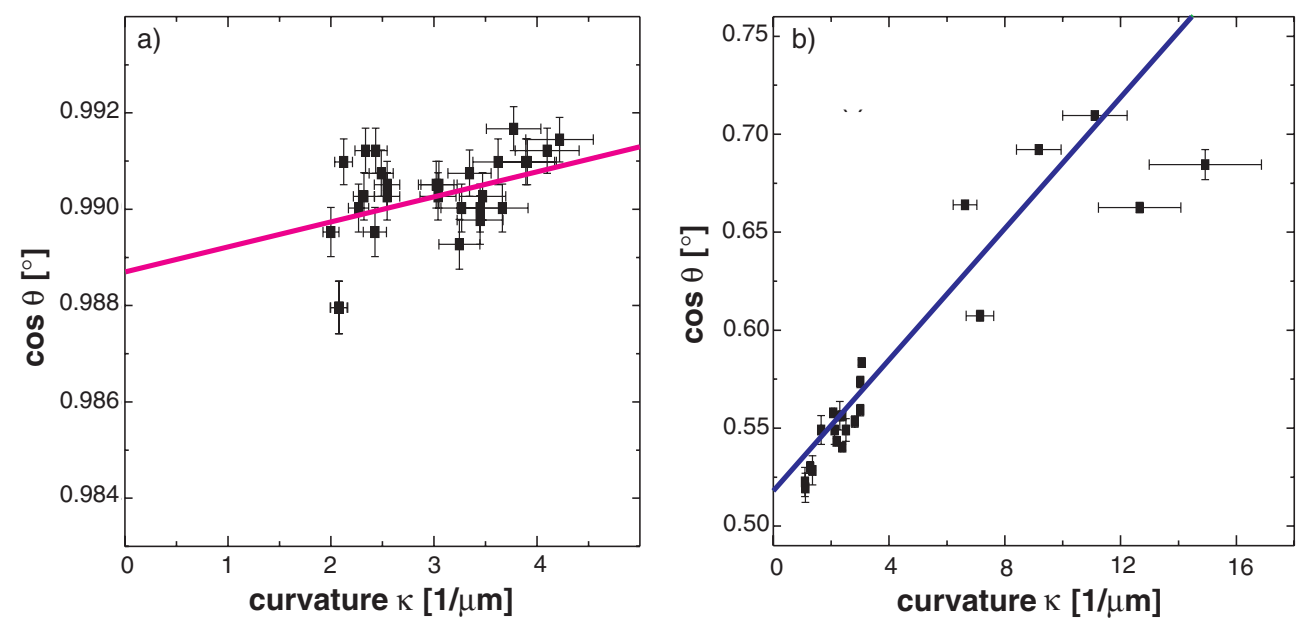

Figure 14. The line tension of PS droplets on different Si substrates. The solid lines show the fitting curves for the experimental data points. (a) An Si substrate with $191 \mathrm{~nm}$ thick oxide. Experimental line tension $v=-1.7(7) \times 10^{-11} \mathrm{~J} \mathrm{~m}^{-1} . v$ is calculated from the effective interface potential derived in [31]: $v=-4(2) \times 10^{-12} \mathrm{~J} \mathrm{~m}^{-1}$. Image (a) is taken from [56]. (b) PS on OTS-Si with $2.4 \mathrm{~nm}$ thick oxide. Experimental $v=-5(2) \times 10^{-10} \mathrm{~J} \mathrm{~m}^{-1} \cdot v$ was calculated from $\phi$ in [31] to be $v=-8(2) \times 10^{-10} \mathrm{~J} \mathrm{~m}^{-1}$.

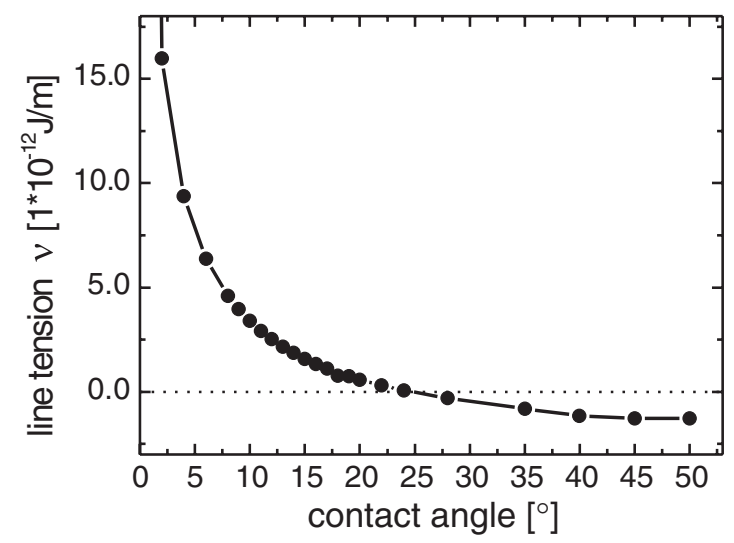

Figure 15. The line tension for PS (droplets) on top of a Si substrate with an oxide layer thickness of $1.75 \mathrm{~nm}$ as a function of the contact angle $\theta$ calculated from the reconstructed effective interface potential in [31].

(cf also [56]) are in good agreement with theory and corroborate also the results for the line tension calculated from the reconstructed effective interface potential [31].

Moreover, these calculations suggest a sign reversal from negative to positive values of the line tension for certain system parameters, as shown in figure 15.

\section{Numerical simulations of dewetting}

The first simulations of dewetting were performed for only a few lattice points $[12,14]$. In the meantime, however, theorists are able to simulate even on three-dimensional arrays with reasonable resolution $[7,21,57-60]$. It is possible nowadays to predict the morphology of 

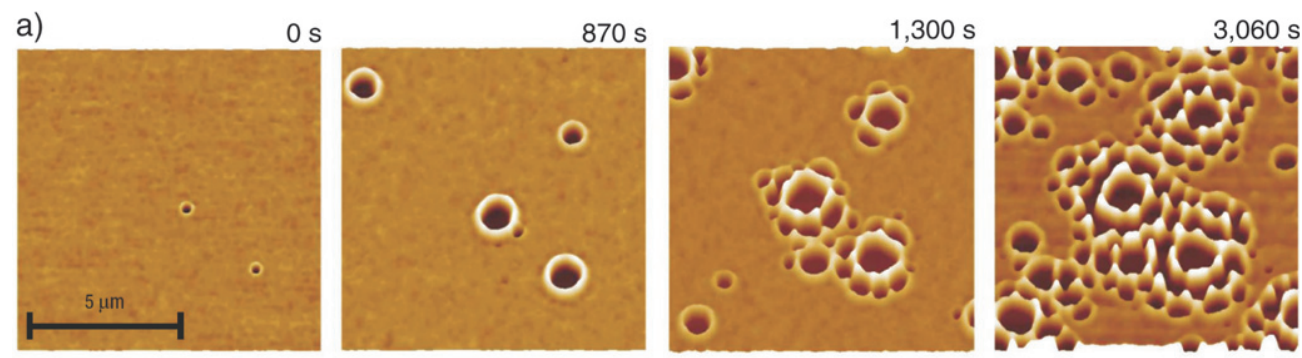

b)
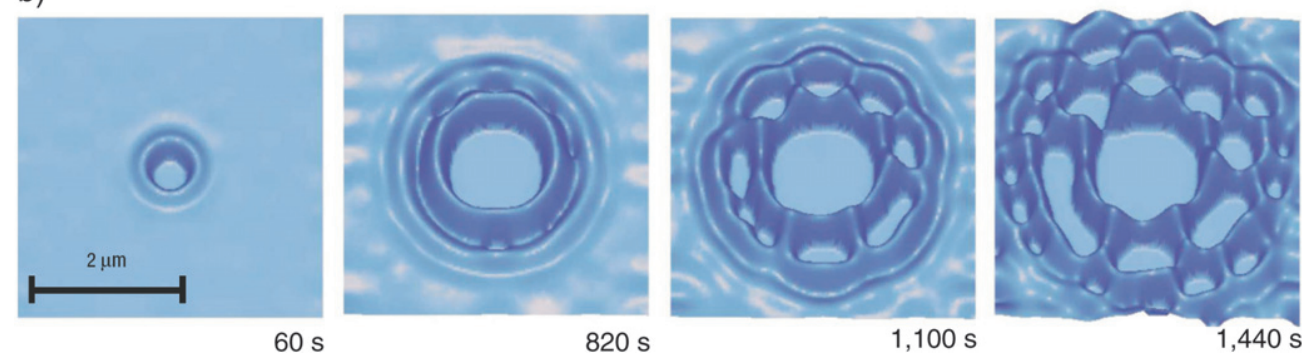

Figure 16. Dewetting morphology of a thin film. These images were taken from [71]. (a) Experiment: a temporal series of AFM scans recorded in situ at $T=70^{\circ} \mathrm{C}$; a $4.9 \mathrm{~nm}$ PS film beads off a wafer with $191 \mathrm{~nm}$ oxide thickness. (b) Simulated dewetting morphology with the same system parameters as in the experiment. The highest points reach $12 \mathrm{~nm}$ above the bottom of the holes. As initial conditions, we took a slightly corrugated film with a depression in its centre.

a dewetting pattern $[7,61]$ and simulate e.g. the evolution of an evaporating film [60]. A quantitative comparison of theoretical and experimental results was not possible however, since the actual intermolecular forces in the experimental system were not precisely known. Instead, for the simulations, some kind of effective interface potential was assumed.

Since it was possible to reconstruct the effective interface potential $\phi$ of the experimental system, it was challenging to perform numerical simulations. This was done in collaboration with theoretical physicists and applied mathematicians [71, 72].

For the simulations, experimental input was given by the reconstructed function $\phi$, as described before, and by the film thickness, surface tension, and viscosity of the liquid film, as known for the experiments. A comparison of the temporal evolution of dewetting in simulation and experiment shows that the experimental process can indeed be reproduced by simulations: the timescale of the simulated film rupture as well as the morphology correspond very well to the experimental data. The comparison of the emerging patterns in experiments and simulations was done with the help of Minkowski functionals [71]. This was a major step in the description of film rupture, creating a powerful tool for studying the impact of various parameters on film rupture, an example of which will be given in the following.

In figure 16, the dewetting scenario of a thin PS film is shown. It exhibits a peculiar morphology which can be understood by looking at its temporal evolution. First, single holes pop up; then, along the perimeter of each hole, secondary and later ternary holes appear.

Upon further experimental and theoretical investigation, it became clear that these 'satellite' holes in fact come from a breakthrough of the trough. A spinodally unstable system strengthens the tendency for the trough to rupture [78]. An identical behaviour was also found by Grün and Becker in their simulations [77]. It should be mentioned here that recent theoretical studies proposed this kind of morphology too, but claimed that certain preconditions 

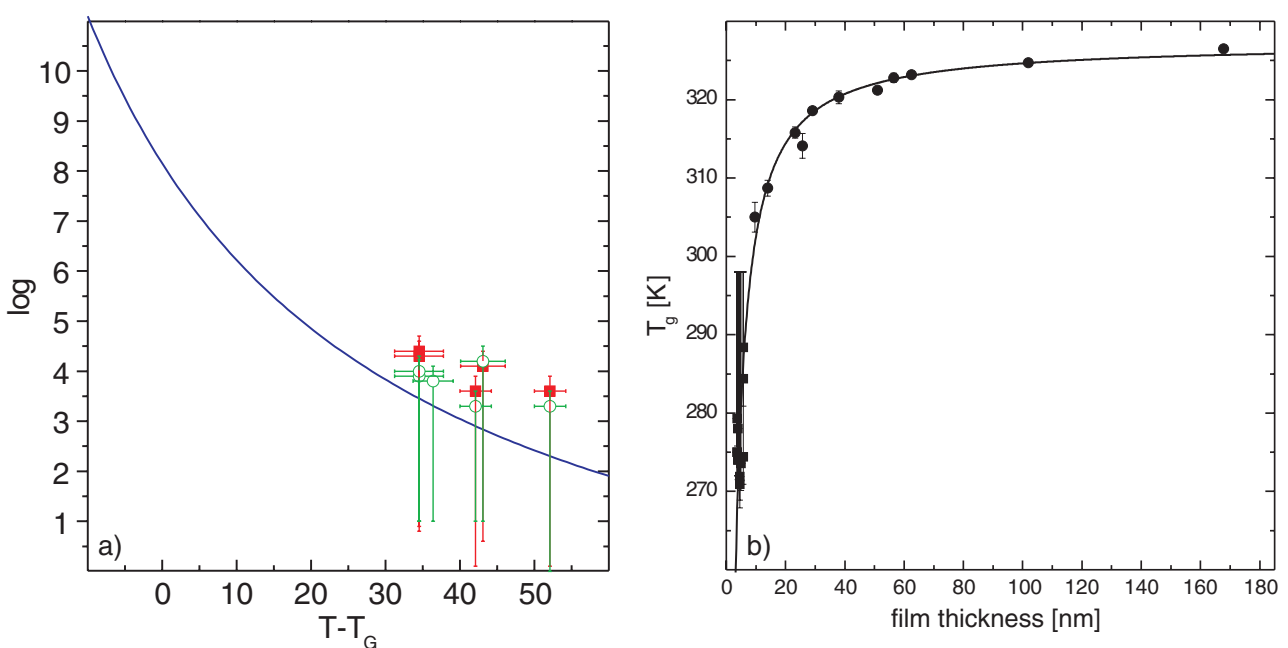

Figure 17. (a) The viscosity of PS(2k) derived from the spinodal break-up time (open circles) and from amplification of the spinodal wavelength (solid squares) for PS film thicknesses in the range from 3.9 to $5 \mathrm{~nm}$. The data points were shifted on the temperature axis according to (b). The solid curve shows a Vogel-Fulcher fit to the bulk viscosity. (b) The glass transition of thin films of PS(2k), as determined from thermal expansion by ellipsometry (circles) and from the growth of spinodal waves (squares). The solid curve represents our model, which has the elastic modulus governing the dominant modes as the only fitting parameter. Picture (b) was taken from [74, 73].

are necessary, e.g. that it is an evaporative system [61] or that the system must be in a metastable state [62]. The experiments, however, demonstrate that neither of these preconditions is necessary and the simulations show that this behaviour should be typical for thin films of Newtonian liquids particularly in the unstable state.

Further studies - simulations and experiments - explored the influence of the shape of the rim profile in the plane of the substrate on the appearance of these satellite holes. The rupturing trough was explored at straight contact lines and in wedge-like geometries where two dewetting contact lines form an angle with each other [72]. There, too, experiments and simulations correspond quite well.

For a quantitative comparison of the timescale in simulation and experiment, the knowledge of the viscosity was very important. Using the bulk viscosity for PS, the timescales of experiment and simulation differed by several orders of magnitude. For a spinodally dewetting system, however, the viscosity can be determined by measuring the rupture time of the film, if the Hamaker constant of the system is known [13]. In our case, the viscosity was significantly reduced as compared to the bulk, which was the motivation for further investigations as described in the next section.

\section{The glass transition temperature of thin films}

The experiments with thin $(<10 \mathrm{~nm})$ short-chained PS films revealed that dewetting may start at room temperature, which is as much as $40 \mathrm{~K}$ below the bulk glass transition temperature $T_{\mathrm{G}}^{0}$. The results of the experiments are shown in figure 17.

For thin supported PS films, several studies, e.g. [63-66], report that $T_{\mathrm{G}}$ decreases with decreasing film thickness. These studies, however, used PS films of molecular weight larger than $100 \mathrm{~kg} \mathrm{~mol}^{-1}$. An explanation of the phenomenon was proposed by de Gennes [70], yet 
it is based on the precondition that the films are thinner than the coil size of the polymer. This condition, however, does not apply to our system: for PS(2k), the radius of gyration is about $1 \mathrm{~nm}$, whereas the film thicknesses examined are typically much larger.

Hence it was not clear whether or not a decrease in $T_{\mathrm{G}}$ accounts for the extremely low viscosities that were measured in the course of the dewetting experiments. It was therefore decided to check $T_{\mathrm{G}}$ for the system under consideration.

$T_{\mathrm{G}}$ can be evaluated by recording the film thickness of a polymer layer as a function of temperature, for instance by ellipsometry. The expansion coefficient of the melt jumps to a higher value as soon as $T>T_{\mathrm{G}}$. Varying the initial thickness of the polymer layer, $T_{\mathrm{G}}(h)$ can be determined. In our case, this was done by ellipsometry and, indeed, a decrease of $T_{\mathrm{G}}$ was found (figure 17(b))

The decrease of $T_{\mathrm{G}}$ for PS(2k) with film thickness corresponds to the behaviour of a longer-chained PS such as PS(100k), and the same fitting functions as proposed in [63$65,67,66,68,69$ ] can be used. de Gennes' theory, however, cannot be valid in the case of PS(2k) for the reasons given above. Therefore, Herminghaus proposed a mode coupling theory approach to explain the behaviour of short- and long-chained polymer films [74, 73, 75], an approach that was recently applied to explain the observed polymer surface melting [76].

Another approach for getting insight into the mobility of thin polymer films is determining the viscosity. Typical rheometers, however, fail since they cannot access very thin films. For spinodally dewetting films the viscosity can be determined via the growth time $\tau$ of the amplitude of the spinodal wavelength: $\tau$ scales linearly with the viscosity $\eta$ as $\tau=\left(48 \pi^{2} \sigma h^{2} \eta\right) / A^{2}$ [13]. If the surface tension $\sigma$, film thickness $h$, and Hamaker constant $A$ are known, the viscosity can be directly inferred by determining $\tau$-for instance by in situ AFM scanning of a spinodally dewetting film (figure 17(a)).

The viscosity of e.g. a $3.9 \mathrm{~nm}$ thick PS(2k) film at $T=53^{\circ} \mathrm{C}$ as determined from $\tau$ is about eight orders of magnitude lower than the bulk viscosity. Such a low viscosity implies also that in these thin films $T_{\mathrm{G}}$ must be lowered. Viscosity and $T_{\mathrm{G}}$ can be related via the Vogel-Fulcher law. Data for $T_{\mathrm{G}}$ that were gained via $\tau$ can now be plotted in the same diagram as the data gained from ellipsometry, completing the latter to smaller film thicknesses. The two data sets can be described by the same fitting function $[74,73,75]$.

For the first time, the viscosity of only $2-5 \mathrm{~nm}$ thick polymer films was experimentally determined. To determine the experimental viscosity of thin, low-molecular-weight PS films therefore seems an important step in enabling quantitative comparison with simulations. On the one hand, molecular dynamic simulations of thin PS(2k) films - corresponding to about 20 monomer units - seem feasible for modern computers [65]. On the other hand, simulations of dewetting films such as the ones shown in [71] need data on the viscosity in order to obtain a realistic timescale that is comparable to that of the experiment.

\section{Conclusion and outlook}

In the framework of the priority programme 'Wetting and Structure Formation at Surfaces and Interfaces', we have studied the stability of thin liquid films on solid surfaces. A detailed analysis of the structures that emerge in the course of dewetting has led to a comprehensive picture of thin film stability. We were for the first time able to distinguish experimentally three different pathways of dewetting (spinodal dewetting as well as homogeneous and heterogeneous dewetting), that were expected theoretically. Studies on spinodally dewetting films allowed us to derive the effective interface potential. Moreover, the relatively simple approach of using additive van der Waals forces turned out to be adequate for correctly describing the potential. Experiments recording the dynamics of dewetting gave insight into 
the viscosity of a thin film. The important result of the experiments was the demonstration that thin films of polystyrene below the entanglement length also exhibit a reduction of the glass transition temperature. Since this case was not included in the common theories explaining the phenomenon, a new theoretical approach was required. We were able to show that mode coupling theory could explain our experimental findings very well.

Both the viscosity and the effective interface potential were important ingredients in the numerical simulations of our co-workers in the priority programme. Moreover, we applied mathematical methods (the Minkowski measures approach) to describe the topographies that emerged in simulations of and experiments on dewetting liquid films. The study provided a quantitative way to compare patterns. As a result we were able to show that experimental and simulated patterns resemble each other strikingly well. In other words, the numerical simulation describe the experimental results in a realistic way and can be used to test the behaviour of a liquid on a hypothetical substrate and to predict stability conditions.

Many questions concerning the dynamical behaviour of liquids on small scales, however, remain unanswered. Future studies will concentrate on the energy dissipation close to the three-phase contact line of a moving front. Can the front profile be used to describe a liquid's properties? Does it reveal whether the liquid is sliding over the surface? Do liquids containing particles or blends of liquids behave similarly? Can we enhance the throughput of lab-on-a-chip devices?

\section{Acknowledgments}

We gratefully acknowledge funding by the German Science Foundation under grant number Ja905/1. Moreover, we would like to thank Siltronic AG, Burghausen, Germany, for the generous supply of silicon wafers and Veeco GmbH, Mannheim, Germany, for the AFM support. We would like to thank Annemarie Saier for her technical help in preparing the films and substrates. We have enjoyed many fruitful discussions with our collaborators in the priority programme and would like to thank all of them for their inspiring contributions.

\section{References}

[1] Young T 1805 Phil. Trans. R. Soc. 95 65-87

[2] Safran S 1994 Statistical Thermodynamics of Surfaces, Interfaces, and Membranes (New York: AddisonWesley)

[3] Israelachvili J 1992 Intermolecular Surface Forces 2nd edn (New York: Academic)

[4] Schick M 1989 Liquids at Interfaces ed J Charvolin et al (Amsterdam: Elsevier Science)

[5] Dietrich S 1988 Phase Transition and Critical Phenomena vol 12, ed C Domb and J L Lebowitz (London: Academic)

[6] de Gennes P G 1985 Rev. Mod. Phys. 57827

[7] Sharma A and Khanna R 1998 Phys. Rev. Lett. 813463

Sharma A and Khanna R 1999 J. Chem. Phys. 1104929

[8] Adamson A W 1990 Physical Chemistry of Surfaces (New York: Wiley)

[9] Hamaker H C 1937 Physica 41058

[10] Lifshitz E M 1956 Sov. Phys._JETP 273 (Engl. Transl.)

[11] Dzyaloshinskii I E, Lifshitz E M and Pitaevskii L P 1961 Adv. Phys. 10165

[12] Vrij A 1966 Discuss. Faraday Soc. 4223

[13] Ruckenstein E and Jain R K 1974 J. Chem. Soc. Faraday Trans. II 70132

[14] Williams M B and Davis S H 1982 J. Colloid Interface Sci. 901

[15] Brochard F and Daillant J 1990 Can. J. Phys. 681084

[16] Mitlin V S 1993 J. Colloid Interface Sci. 156491

[17] Mitlin V S 1994 Colloids Surf. A 8997

[18] e.g. Blossey R 1995 Int. J. Mod. Phys. B 93489

[19] Frumkin A N 1938 J. Phys. Chem. USSR 12337 (This reference has been brought to our attention by V Mitlin, March 2001) 
[20] Jacobs K 1997 Stabilität und Dynamik flüssiger Polymerfilm (Allensbach: UFO Verlag) ISBN 3-930803-10-0

[21] Konnur R, Kargupta K and Sharma A 2000 Phys. Rev. Lett. 84931 and references therein

[22] Mecke K R 1994 Integralgeometrie in der Statistischen Physik-Perkolation, Komplexe Flüssigkeiten und die Struktur des Universums, Reihe Physik Bd 25 (Frankfurt a.M.: Verlag Harri Deutsch)

[23] Herminghaus S, Jacobs K, Mecke K, Bischof J, Fery A, Ibn-Elhaj M and Schlagowski S 1998 Science 282916

[24] Jacobs K, Mecke K and Herminghaus S 1998 Langmuir 14965

[25] Jacobs K, Seemann R and Mecke K 2000 Statistical Physics and Spatial Statistics ed K Mecke and D Stoyan (Heidelberg: Springer)

[26] Reiter G 1992 Phys. Rev. Lett. 6875

[27] Reiter G 1993 Langmuir 91344

[28] Croll S G 1979 J. Appl. Polym. Sci. 23847

[29] Podzimek D, Seemann R, Herminghaus S, Mecke K and Jacobs K 2005 in preparation

[30] Bischof J, Scherer D, Herminghaus S and Leiderer P 1996 Phys. Rev. Lett. 771536

[31] Seemann R, Herminghaus S and Jacobs K 2001 Phys. Rev. Lett. 865534

[32] Seemann R, Herminghaus S and Jacobs K 2001 J. Phys.: Condens. Matter 134925

[33] de Gennes P-G 1979 C. R. Acad. Sci. B 228219

[34] Redon C, Brzoka J B and Brochard-Wyart F 1994 Macromolecules 27468

[35] Brochard F, de Gennes P G, Hervert H and Redon C 1994 Langmuir 101566

[36] Brochard F, di Meglio J-M and Quere D 1987 C. R. Acad. Sci. II 304553

[37] Brochard F, Redon C and Sykes C 1992 C. R. Acad. Sci. II 31419

[38] Reiter G, Auroy P and Auvray L 1996 Macromolecules 292150

[39] Reiter G and Khanna R 2000 Langmuir 166351

[40] Jacobs K, Seemann R, Schatz G and Herminghaus S 1998 Langmuir 144961

[41] Safran S A and Srolovitz D J 1986 Europhys. Lett. 261

[42] Ghatak A, Khanna R and Sharma A 1999 J. Colloid Interface Sci. 212483

[43] Srolovitz D J 1993 personal communication

[44] Seemann R, Herminghaus S and Jacobs K 2001 Phys. Rev. Lett. 87196101

[45] Lord Rayleigh F R S 1878 Proc. Lond. Math. Soc. 104

[46] Quéré D 2004 Nat. Mater. 379

[47] Checco A, Guenoun P and Daillant J 2003 Phys. Rev. Lett. 91186101

[48] Harkins W D 1937 J. Chem. Phys. 5135

[49] Churaev N V, Starov V M and Derjaguin B V 1982 J. Colloid Interface Sci. 8916

[50] Rowlinson J S and Widom B 1984 Molecular Theory Capillarity (New York: Oxford Science) p 240

[51] Getta T and Dietrich S 1998 Phys. Rev. E 57655

[52] Li D and Neumann A W 1990 Colloids Surf. 43195

[53] Amirfazli A, Kwok D Y, Gaydos J and Neumann A W 1998 J. Colloid Interface Sci. 2051

[54] Pompe T and Herminghaus S 2000 Phys. Rev. Lett. 851930

[55] Pompe T 2002 Phys. Rev. Lett. 89076102

[56] Seemann R, Jacobs K and Blossey R 2001 J. Phys.: Condens. Matter 134915

[57] Sharma A and Jameel A 1993 J. Colloid Interface Sci. 161190

[58] Khanna R and Sharma A 1997 J. Colloid Interface Sci. 19542

[59] Oron A, Davis S H and Bankoff S G 1997 Rev. Mod. Phys. 69931

[60] Oron A 2000 Phys. Rev. Lett. 852108

[61] Kargupta K, Konnur R and Sharma A 2001 Langmuir 171294

[62] Thiele U, Velarde M G and Neuffer K 2001 Phys. Rev. Lett. 87016104

[63] Keddie J L, Jones R A L and Cory R A 1994 Europhys. Lett. 2759

[64] Mattsson J, Forrest J A and Börgesson L 2000 Phys. Rev. E 625187

[65] Torres J A, Nealey P F and de Pablo J J 2000 Phys. Rev. Lett. 853221

[66] Forrest J A and Dalnoki-Veress K 2001 Adv. Colloid Interface Sci. 94167 and references therein

[67] Dalnoki-Veress K, Forrest J A, de Gennes P-G and Dutcher J R 2000 J. Physique Coll. IV 10221

[68] Teichroeb J H and Forrest J A 2003 Phys. Rev. Lett. 91016104

[69] Ellison C J and Torkelson J M R 2003 Nat. Mater. 2695

[70] de Gennes P-G 2000 Eur. Phys. J. E 2201 de Gennes P-G 2000 C. R. Acad. Sci. 1/IV 1179

[71] Becker J, Grün G, Seemann R, Mantz H, Jacobs K, Mecke K R and Blossey R 2002 Nat. Mater. 259

[72] Neto C, Jacobs K, Seemann R, Blossey R, Becker J and Grün G 2003 J. Phys.: Condens. Matter 153355 Neto C, Jacobs K, Seemann R, Blossey R, Becker J and Grün G 2003 J. Phys.: Condens. Matter 15 S421

[73] Herminghaus S, Jacobs K and Seemann R 2003 Eur. Phys. J. E 12101 
[74] Herminghaus S, Jacobs K and Seemann R 2001 Eur. Phys. J. E 5531

[75] Herminghaus S 2002 Eur. Phys. J. E 8237

[76] Herminghaus S, Landfester K and Seemann R 2004 Phys. Rev. Lett. 93017801

[77] Becker J 2004 Thesis University of Bonn

[78] Herminghaus S, Jacobs K and Seemann R 2002 Phys. Rev. Lett. 89056101

[79] Neto C, Münch A, Seemann R and Jacobs K 2005 in preparation

[80] Neto C and Jacobs K 2004 Physica A 33966 